\title{
Acknowledgement to Reviewers of Journal of Functional Morphology and Kinesiology in 2016
}

\author{
Journal of Functional Morphology and Kinesiology Editorial Office \\ MDPI AG, St. Alban-Anlage 66, 4052 Basel, Switzerland; jfmk@mdpi.com \\ Published: 11 January 2017
}

The editors of Journal of Functional Morphology and Kinesiology would like to express their sincere gratitude to the following reviewers for assessing manuscripts in 2016.

We greatly appreciate the contribution of expert reviewers, which is crucial to the journal's editorial process. We aim to recognize reviewer contributions through several mechanisms, of which the annual publication of reviewer names is one. Reviewers receive a voucher entitling them to a discount on their next MDPI publication and can download a certificate of recognition directly from our submission system. Additionally, reviewers can sign up to the service Publons (https://publons.com) to receive recognition. Of course, in these initiatives we are careful not to compromise reviewer confidentiality. Many reviewers see their work as a voluntary and often unseen part of their role as researchers. We are grateful to the time reviewers donate to our journals and the contribution they make.

If you are interested in becoming a reviewer for Journal of Functional Morphology and Kinesiology, see the link at the bottom of the webpage http://www.mdpi.com/reviewers.

The following reviewed for Journal of Functional Morphology and Kinesiology in 2016:

Alini, Mauro
Armstrong, Thomas
Borgo, Andrea
Castorina, Sergio
Cotter, Joshua A.
Di Corrado, Donatella
Dickin, Clark
Fairman, Ciaran
Ferreira Mendes, Alexandrina
Harder, Helena
Imbesi, Rosa
Koldenhoven, Rachel M.
Leiter, Jeff R. S.
Lopomo, Nicola Francesco
Paoli, Antonio
Pichler, Karin
Ransdell, Lynda
Silva, Luís
Stefani, Laura

Anifandis, George
Assanelli, Deodato
Caff, Giuseppe
Castrogiovanni, Paola
D'Amora, Ugo
Di Giunta, Angelo
Eltoukhy, Moataz
Fantuzzi, Guglielmina
Glenn, Thomas C.
Hart, Kathryn H.
Katzman, Wendy
Kontoyianni, Meropi
Li Volti, Giovanni
Madry, Henning
Pavone, Vito
Pike, Elizabeth
Rasmussen, Peter
Sobhani, S.
Stoddart, Martin

Anifandis, George

Caff, Giuseppe

D'Amora, Ugo

Di Giunta, Angelo

Fantuzzi, Guglielmina

Hart, Kathryn H.

man, Wendy

Li Volti, Giovanni

Madry, Henning

one, Vito

Stoddart, Martin
Arco, Alba

Bertollo, Maurizio

Callewaert, Bert

Coco, Marinella

De Luca, Alessio

Di Marco, Robertp

Ermolao, Andrea

Feigenbaum, Luis A.

Guglielmino, Claudia

Hue, Olivier

Klika, Riggs

Lauche, Romy

Liguori, Giorgio

Morland, Cecilie

Perciavalle, Vincenzo

Prescher, Andreas

Rodella, Luigi Fabrizio

Sorci, Guglielmo

Szychlinska, Marta Anna 
Taylor, William R.

Trovato, G.M.

Tsunoda, Kenji

Watt, Peter
Torres, Rui

Trovato, Guglielmo

Uga, Daisuke

Workman, Victoria
Trovato, Francesca Maria

Tsonev, Svetlin

Wang, Lingyan

(c)

(C) 2017 by the authors. Submitted for possible open access publication under the terms and conditions of the Creative Commons Attribution (CC-BY) license (http://creativecommons.org/licenses/by/4.0/). 